\title{
Gibt es Wege aus der selbstverschuldeten Irrelevanz des qualitativen Offstreams ${ }^{1}$
}

\section{Norbert Groeben}

\section{Keywords:}

Gegenstands-

Methodik-

Interaktion, qua-

litatives vs.

quantitatives

Paradigma,

Binnen- und

Außenkonkurrenz,

integrativer

Pluralismus,

Schichten-

ontologie,

esoterische

Kunstlehren,

Mixed Methods-

Ansätze, komplexe statistische Auswertungsmodelle
Zusammenfassung: Die weitgehende Irrelevanz des qualitativen Paradigmas in der psychologischen Forschung wie Lehre kommt nicht nur durch das Hegemonialstreben des quantitativen Paradigmas zustande. Bedingt durch das Prinzip der Anpassung der Methodik an den Gegenstand liegt auch eine Zersplitterung der qualitativen Richtung vor, die sich vor allem im Fehlen eines systematischen Methoden-Begriffs manifestiert. Die Überzeugung, die bessere Psychologie zu treiben, führt zu destruktiver Konkurrenz innerhalb des qualitativen wie in Relation zum quantitativen Paradigma. Stattdessen sollten integrative, z.B. schichtenontologische Ansätze zur Verbindung von qualitativen und quantitativen Forschungsmethoden einschließlich einer Abgrenzung von esoterischen Kunstlehren verfolgt werden. Insbesondere bietet die Entwicklung komplexer statistischer Auswertungsverfahren einen unmittelbar wirksamen Weg zur Überwindung der selbstverschuldeten Irrelevanz des qualitativen Forschungsansatzes.

Einführung zu diesem Beitrag von Margrit Schreier und Franz Breuer

Inhaltsverzeichnis

1. Das quantitative Paradigma als homogener, hegemonialer Mainstream

2. Die weit gehende Irrelevanz des qualitativen Paradigmas

3. Die Selbstverschuldetheit der qualitativen Irrelevanz

4. Wege aus der selbstverschuldeten Irrelevanz

4.1 Integrativer Theorienpluralismus

$\underline{4.2}$ Integrative qualitativ-quantitative Versuchsplanung

$\underline{4.3}$ Ausarbeitung komplexer statistischer Auswertungsansätze

Danksagung

Literatur

Zum Autor

Zitation

\section{Das quantitative Paradigma als homogener, hegemonialer Mainstream}

Das Thema, das ich behandeln möchte, setzt über das Kontrastprinzip des Assoziierens, Schlussfolgerns etc. voraus, dass es in der Psychologie eine irgendwie geartete Form von Mainstream gibt. Interessanterweise ist diese Voraussetzung aber wissenschaftstheoretisch keineswegs unumstritten. Wenn man 'Mainstream'2 als wissenschaftssoziologische Benennung dessen ansieht, was wissenschaftstheoretisch seit KUHN $(1967,1972)$ 'Paradigma' oder

1 Dieser Beitrag ist eine überarbeitete Fassung eines Vortrages während des $\underline{2}$. Berliner Methodentreffens Qualitative Forschung im Juli 2006 an der Freien Universität Berlin.

2 In diesem Beitrag werden objektsprachliche Sprachelemente innerhalb von metasprachlichen Aussagen durch einfache Anführungsstriche markiert. 
'disziplinäre Matrix' genannt wird, dann geht es also darum, ob es in der Psychologie einen beherrschenden Forschungs-/Theorienansatz gibt oder nicht. Und manche Wissenschaftstheoretiker/innen bzw. -historiker/innen argumentieren hier so, dass die Psychologie insgesamt (z.B. im Gegensatz zur Physik) noch in einem vorparadigmatischen Zustand sei, also noch gar nicht so präzise Theorieausarbeitungen vorgelegt habe, dass man irgendeiner davon einen Paradigmastatus zuerkennen könnte (vgl. GROEBEN \& WESTMEYER 1981; HERRMANN 1994; vgl. transdisziplinär: FRANCK 2002; SCHURZ 1988). [1]

Zugleich gibt es in der alltäglichen Wissenschaftspraxis eine Fülle von Erfahrungen, die dafür sprechen, dass es wissenschaftssoziologisch und -psychologisch auch in der Psychologie - seit jeher - bestimmte beherrschende Theorietraditionen gegeben hat und gibt, und zwar letztlich unabhängig davon, ob ein der Physik vergleichbarer Explizitheits- und Formalisierungsgrad der Theorieelaboration erreicht ist oder nicht. Am deutlichsten im historischen Bewusstsein ist diesbezüglich sicher die Phase des Behaviorismus verankert, aber auch für die nachfolgende Ära des Kognitivismus darf man wohl mit Fug und Recht von einer Hauptströmung sprechen, die sich interdisziplinär als Kognitionswissenschaft (Cognitive Science mit der Computer-Metapher des menschlichen Geistes) versteht, intradisziplinär in der Psychologie als Informationsverarbeitungsansatz an- und auftritt (vgl. ERB 1997; GROEBEN 2003; LÜCK 1996). Dabei sind die relevanten wissenschaftspsychologischen und -soziologischen Manifestationen und Dynamiken solcher Hauptströmung(en) deutlich weniger systematisch erforscht als die wissenschaftstheoretischhistorische Dimension. Insofern werden meine Reflexionen nolens volens vor allem auf Beispiele von subjektiven Altagserfahrungen aus der Wissenschaftspraxis zurückgreifen müssen, für die ich aber einen gewissen Wiedererkennenswert bei anderen Mitgliedern der Scientific Community erhoffe. [2]

Dabei gibt es z.T. eine aparte paradoxale Verschränkung der Ebenen. In der Alltagspraxis ist nämlich nicht selten die wissenschaftstheoretische Negierung eines (beherrschenden) Paradigmas in der Psychologie der Indikator dafür, dass gerade dieses vertreten wird. Das Argument lautet: 'Ich kenne keinen herrschenden Ansatz, ich kenne nur gute oder schlechte Wissenschaft!' Wundersamer Weise zeigt sich dann aber schnell, dass gute Wissenschaft prototypisch experimentell sein muss, auf jeden Fall die interne Validität von Untersuchungen zu sichern hat, was im Zweifelsfall die Nicht-Berücksichtigung von subjektivem Erleben, individueller Geschichtlichkeit etc. beim ErkenntnisObjekt rechtfertigt, d.h. die nomothetische Perspektive ganz eindeutig gegenüber der idiographischen präferiert, Verhaltens- gegenüber Erlebensdimensionen den Vorzug gibt und schließlich inhaltlich kognitiv ausgerichtet ist: im Sinne der Naturalisierung des Mentalen, sowohl was die grundlegende ontologische Frageperspektive als auch die interdisziplinäre Fundierungsrelation angeht. Damit aber haben wir die wichtigsten prototypischen Merkmale des quantitativen Paradigmas in der Psychologie versammelt, so dass - ohne dies hier weiter auszudifferenzieren - als Ausgangspunkt festgehalten werden kann: Es gibt inn doch, den Mainstream in der Psychologie. [3] 
Dabei ist es psychologisch nicht sonderlich verwunderlich, dass eine solche Richtung von außen kompakter wahrgenommen wird als von innen. Insbesondere in Zeiten des neoliberalen Konkurrenzdrucks auch in der Wissenschaft treibt die Vertreter/innen des Mainstreams naturgemäß zunächst einmal die Durchsetzung gegenüber den unmittelbaren Theoriealternativen innerhalb des eigenen Paradigmas um; denn diese gilt es hinter sich zu lassen, um z.B. im Bereich der Drittmitteleinwerbung möglichst erfolgreich zu sein (was, nebenbei bemerkt, im Rahmen der herrschenden Wachstumsideologie heißt: immer erfolgreicher zu sein, d.h. Stagnation ist Rückschritt!). Zugleich führt diese Binnenkonkurrenz allerdings zu dem indirekten und damit letztlich noch stärkeren Effekt der Außenabgrenzung. Denn die Binnenkonkurrenz ist nur durch die möglichst optimale Erfüllung der paradigmaspezifischen Zielkriterien zu entscheiden; und das sind im quantitativen Paradigma nun einmal methodologische Kriterien. Daraus resultiert die fast zwanghafte Rede von den methodologischen 'Standards', in der aus externer Sicht die (prinzipielle) Anpassung des Gegenstands an die Methodik zum Ausdruck kommt. Mit den methodologischen Standards ist aber nach außen auch eine außerordentlich starke Hegemonialität verbunden (MÉTRAUX 1985): Alles, was diesen Standards nicht entspricht, wird als nicht förderungswürdig bewertet, implizit tendenziell sogar als nicht (genügend) wissenschaftlich (TOULMIN \& LEARY 1985). Manifest und beobachtbar wird diese Hegemonialität z.B. immer in interdisziplinären Gutachtensgruppen zusammen mit anderen Sozial- und Geisteswissenschaften (von der Pädagogik über Kommunikations-/Medienwissenschaft bis zur Linguistik etc.). In all solchen Konstellationen versuchen die psychologischen Mitglieder quasi automatisch, die Grenzen zum Bereich der Unwissenschaftlichkeit bzw. zumindest wissenschaftlichen Marginalität (und damit Nicht-

Förderungswürdigkeit) zu bestimmen - übrigens in der Regel mit Erfolg. [4]

\section{Die weit gehende Irrelevanz des qualitativen Paradigmas}

Bei aller Binnenkonkurrenz ist der Mainstream des quantitativen Paradigmas (wissenschaftpsychologisch/-soziologisch) also außerordentlich homogen und hegemonial, d.h. versucht, Nebenströmungen noch weiter zu marginalisieren, approximativ zu eliminieren. Dabei wird diese Marginalisierung, entsprechend der eingangs angeführten Leugnung, dass es überhaupt so etwas wie Haupt- und Nebenströmung gibt, selbstverständlich nicht explizit und offen betrieben. Die seit Jahrzehnten eingesetzte Strategie ist vielmehr, als Lippenbekenntnis eine Methodenvielfalt nicht nur zu tolerieren, sondern aktiv zu propagieren; lediglich sind manche Methoden eben nur im Rahmen von Erkundungsstudien etc., d.h. als Heuristik einsetzbar. Damit wird dem qualitativen Paradigma allerdings abgesprochen, ein (gleichberechtigter) Weg zum Erkenntnisgewinn im Sinne der Realitätsprüfung von Theorien, Hypothesen, Modellen zu sein. Was konzediert wird, ist das heuristische Aufstellen von Hypothesen, die dann innerhalb des quantitativen Paradigmas auf Validität zu prüfen sind. Im Rahmen der dabei zugrunde gelegten wissenschaftstheoretischen Kategorisierung aber ist das Aufstellen von Hypothesen ein vorwissenschaftlicher Akt, erst die systematische Validitätsprüfung dieser Hypothesen stellt einen wissenschaftlichen Erkenntnisgewinn dar. So wandelt sich unter der Hand die scheinbare Liberalität 
des Methodenpluralismus schnell in das Dogma von der Vor- und damit Unwissenschaftlichkeit der qualitativen Methoden. Das bedeutet nicht, dass diese trickreiche Marginalisierungsstrategie von den Vertretern/innen des quantitativen Mainstreams bewusst eingesetzt wird; nein, die meisten lernen diese Strategie im Rahmen ihrer szientifisch-quantitativen Sozialisation und wenden sie als Automatismus an - mit dem erfreulichen (Neben-) Ergebnis, die ungeliebten qualitativen Methoden damit aus dem Bereich des ernsthaft zu Studierenden, der aufzuarbeitenden Wissenschaftlichkeit ausgegrenzt zu haben. [5]

Das ist dann auch die Diskrepanz, die Widersprüchlichkeit, die von den Mitgliedern, Vertretern/innen des qualitativen Paradigmas erfahren wird. Im Prinzip ein Double bind. Explizit, mit großem Nachdruck: Ihr gehört dazu, zur Psychologie! Implizit, mit großer Konsequenz: Ihr gehört nicht dazu, zur Wissenschaft! Mit diesem pragmatischen Paradox gelingt es dann dem quantitativen Paradigma als Hauptstrom, das qualitative zu marginalisieren, in Forschung wie Lehre relativ irrelevant zu halten. Diese wissenschaftssoziologische und -psychologische Irrelevanz ist, wie erwähnt, m.W. noch nicht systematisch empirisch nachgewiesen. Aber es gibt durchaus aussagekräftige Indikatoren. Im Bereich der Forschung handelt es sich dabei z.B. um das Problem, dass aus Sicht des qualitativen Paradigmas dadurch, dass ausschließlich die Deutsche Gesellschaft für Psychologie in die Wahl der Fachgutachter/innen bei der DFG involviert ist, zu wenige Gutachter/innen mit Kenntnissen und Kompetenzen in qualitativer Methodik gewählt werden. Die Zulassung weiterer Gesellschaften (z.B. der Neuen Gesellschaft für Psychologie) scheitert aber daran, dass es sich hier nicht um rein wissenschaftliche Gesellschaften (im Sinne der Forschungsorientierung) handelt, weil auch Praktiker/innen als Mitglieder zugelassen sind. Einschlägige Bemühungen zur Erweiterung des Begutachtungsverfahrens der DFG in Richtung auf eine Institutionalisierung von Teilhabe des qualitativen Paradigmas sind deshalb bisher immer noch gescheitert. Im Bereich der Lehre dürfte die weitgehende Irrelevanz des qualitativen Paradigmas relativ augenfällig sein, wenn man sich das Methodencurriculum in Psychologie anschaut: von der frühren Rahmenstudienordnung für das Einheitsdiplom bis hin zu den einzelnen Studiengängen an den deutschen Universitäten und die neuesten Bachelor-/Masterentwürfe. Qualitative Methoden-Veranstaltungen als Pflicht-/Leistungskurse sind dabei extrem selten. Und dort, wo sie existieren (z.B. in Köln die Übung 'Qualitative Datenerhebung und -auswertung'), sind sie auch in stetiger Regelmäßigkeit Angriffen von Seiten quantitativ orientierter Fachvertreter/innen ausgesetzt. Das Fazit scheint berechtigt: Das qualitative Paradigma erfreut sich in der BRD in Forschung wie Lehre einer weitgehenden Irrelevanz. [6]

Nun kann das die Vertreter/innen des quantitativen Paradigmas freuen, für das qualitative dürfte es eher ein Anlass zu Frustration und zum Bemühen um Änderung bedeuten. Soll der Versuch, diese Irrelevanz auf Dauer zu beheben, erfolgreich sein, so wird er allerdings von zureichenden Erklärungen des IstZustandes ausgehen müssen. Das heißt, wir müssen fragen: Wieso ist es eigentlich bisher dem qualitativen Paradigma nicht oder so wenig gelungen, sich aus dieser Irrelevanz zu befreien? Dabei gibt es die eine oder andere Erklärung, 
die zwar historisch relevant ist, sich aber heute kaum noch auswirken dürfte. Dazu gehört in erster Linie, dass sich gerade die geisteswissenschaftliche Psychologie zur Zeit des Nationalsozialismus völlig desavouiert hat, indem sie sich selbst mit maximaler Anbiederung 'gleichgeschaltet' hat (vgl. GEUTER 1986; GRAUMANN 1985). Dieses Faktum hat die Entwicklung des qualitativen Paradigmas in Deutschland nach dem Krieg sicher erheblich beeinträchtigt. Eine Konsequenz war, dass qualitative Methoden ganz grundsätzlich erst wieder zumindest diskussionswürdig wurden, als sie aus den USA (re-)importiert wurden. Dies aber geschah in den 1960er und 70er Jahren, so dass nach den weiteren Gründen dafür zu fragen ist, warum sich auch nach 1980 das qualitative Paradigma nicht aus der weitgehenden Irrelevanz gegenüber dem quantitativen befreien konnte. [7]

\section{Die Selbstverschuldetheit der qualitativen Irrelevanz}

Hier ist sicherlich zunächst einmal die komplementäre Perspektive zur Homogenität und Hegemonialität des quantitativen Paradigmas anzuführen.

Diese Homogenität kommt auf höchstem Abstraktionsniveau, wie skizziert, nicht zuletzt durch das Prinzip Anpassung des Gegenstands an die Methodik zustande. Das qualitative Paradigma ist nun aber durch den Gegenpol gekennzeichnet, das Prinzip der größtmöglichen Anpassung der Methodik an den Gegenstand (vgl. z.B. JÜTTEMANN 1983, 1992), mit der komplementären Konsequenz einer sehr großen Heterogenität. Im Prinzip gibt es nicht den einen Offstream, sondern eine Fülle von Nebenströmungen: von der phänomenologischen Psychologie bis zur Ethnomethodologie, von der objektiven Hermeneutik bis zur Diskursanalyse, von der Morphologie bis zur narratologischen Psychologie, von der Kulturpsychologie bis zur DialogHermeneutik, von der Grounded Theory bis zur Triangulation, von der verstehenden Verweisungsanalyse (LAUCKEN 1989) bis zum Integrationsversuch von Monismus und Dualismus (GROEBEN 1986) etc. (vgl. z.B. FLICK, KARDOFF, ROSENSTIEL \& WOLFF 1991; KÖNIG \& ZEDLER 1995; LAMNEK 1988, 1989). Und diese Heterogenität wird zur Zersplitterung, indem jede Richtung auch noch ihr eigenes Gegenstandsvorverständnis kultiviert und gegen andere Richtungen abschottet. Denn die Anpassung der Methodik an den Gegenstand kann bekanntermaßen nicht so gedacht werden, dass 'der' Gegenstand für alle gleichermaßen eindeutig vorfindlich ist, sondern es handelt sich immer um ein Gegenstandsvorverständnis, mit dem an dem komplexen psychologischen Gegenstand die interessierenden, relevanten Merkmale abgehoben werden (GROEBEN 1986, S.49ff.). Damit sind ontologischanthropologische Annahmen impliziert, wie sie sich auch für Erklärungskonzepte (Erklärungstypen nach der Terminologie von GROEBEN 1999) explizieren lassen: So muss die Psychoanalyse z.B. bei Versprechern nach unbewussten Motiven suchen und diese finden, um eine adäquate Erklärung zu konstatieren (GRÜNBERG 1988), während die Linguistik semantisch-pragmatische Sprachähnlichkeiten (zwischen dem geplanten und dem geäußerten Sprechakt) als zureichende Erklärungsursachen ansetzt (und damit die These der unbewussten Verursachung von Versprechern explizit negiert) (LEUNINGER 1993, 1996). Was resultiert, ist eine fast amorphe Vielfalt sowohl auf der 
Gegenstands- als auch auf der Methodik-Seite im qualitativen Paradigma. Damit aber fehlt es an der auch nur minimalen Kohärenz, die Voraussetzung dafür wäre, eine irgendwie geartete gemeinsame Gegenposition zum herrschenden quantitativen Paradigma aufzubauen. Die Marginalisierung des qualitativen durch das quantitative Paradigma, so habe ich oben argumentiert, läuft vom quantitativen Mainstream aus gesehen praktisch nebenbei ab, nicht einmal planvoll intendiert, sondern als gern mitgenommener Nebeneffekt der internen Binnenkonkurrenz. Dieser Erfolg kommt aber letztlich im vorliegenden Umfang der forschungs- und lehrmäßigen Irrelevanz des qualitativen Paradigmas nur dadurch zustande, dass dieses erheblich zur eigenen Irrelevanz beiträgt: durch Zersplitterung auf Gegenstands- wie Methodenebene! [8]

Eine konkurrenzfähige Alternative zum quantitativen Hauptstrom müsste sich m.E. auf möglichst vielen Dimensionen zu einer kohärenten, konsensfähigen Grundstruktur durchringen. Sicher ist ein theoretischer und methodischer Pluralismus innerhalb des qualitativen Paradigmas unverzichtbar; ein Pluralismus, der auch noch möglichst reflektiert, wissenschaftstheoretisch differenziert und problembewusst sein sollte. Nur im Hauptstrom kann man es sich erlauben, monolithische und z.T. von der Diskussion überholte wissenschaftstheoretische Positionen einfach als allgemein akzeptiert vorauszusetzen, ohne sie weiter begründen zu müssen; das Einverständnis der überwältigenden Mehrheit ist in der Regel (auch in der Wissenschaft) Begründung genug. Die Minderheitsposition dagegen unterliegt immer einer besonderen Begründungspflicht. Man mag das als ungerecht empfinden, es hat aber auch den großen Vorteil, dass man zu einem differenzierteren Problembewusstsein und Kenntnisstand gezwungen wird. Allerdings nur, solange man sich offensiv in die argumentative Auseinandersetzung mit der Mehrheitsposition hineinbegibt. Dazu aber ist neben der pluralistischen Grundhaltung auch die Anstrengung der integrativen Elaboration nötig; d.h. der Ausarbeitung einer die verschiedenen Nebenströmungen einigenden Grundstruktur. Dieser Anstrengung aber unterzieht sich bisher der Offstream m.E. mitnichten. Im Gegenteil: Jede Teilströmung arbeitet mehr oder weniger daran, den eigenen qualitativen Ansatz als den allein oder doch zumindest primär selig machenden Weg zu etablieren. Dabei werden dann durchaus die gleichen Sekundärstrategien der Positionsdurchsetzung eingesetzt, wie sie am Mainstream und seinem Hegemonialstreben so vehement beklagt werden: Zitationskartelle, Totschweigen alternativer Positionen, egoistische Nutzung von Gate keeper-Funktionen etc. (vgl. schon WEINGART 1972; s. auch FRÖHLICH 1999) Es ist eben in der Wissenschaft so wie im Leben generell auch: Die Benachteiligten sind nicht automatisch die besseren Menschen, gehen miteinander nicht unbedingt rationaler, fairer um im Vergleich zu den Mächtig(er)en. [9]

Das Aparte ist, dass in diesem Fall allerdings die Strafe auf dem Fuße folgt. Denn solches sich gegenseitig Bekämpfen, Konkurrieren statt Integrieren, schwächt natürlich den Nebenstrom zusätzlich. Es hat praktisch zu einer Auflösung des Methoden-Begriffs im qualitativen Paradigma geführt. Wobei es aus meiner Sicht auch an der Motivation fehlt, diesen Zustand, der die Irrelevanz des qualitativen Offstreams nur zementieren kann, wirklich zu ändern. Als 
zentrale Gründe für diesen Unwillen zur Änderung sehe ich drei Dynamiken: Zunächst einmal wird von manchen Vertretern/innen des qualitativen Ansatzes befürchtet, dass eine solche Annäherung an den quantitativen Mainstream qua Systematisierung des Methoden-Konzepts den eigenen Ansatz korrumpiert, zu sehr angleicht; dies läuft auf eine Argumentation in Richtung einer 'reinen Lehre' hinaus, was letztlich als dogmatisch zu kritisieren ist. Die zweite Dynamik kreist um die Funktion einer 'positiven Marginalität', eine Position, die mir sehr sympathisch ist; denn ich denke auch, dass Veränderungen nur durch Außenseiter zustande kommen, die diese Position als eine positiv gewollte, konstruktive wählen und einnehmen wollen (vgl. UNGER 2000). Aber Veränderung impliziert auch hier Kommunikation, Auseinandersetzung, die selbstverständlich rational, integer, kreativ sein sollte. Auseinandersetzung mit der Mehrheitsposition heißt nicht, sich im Stil anzugleichen, sondern im Gegenteil, den in der positiven Marginalität entwickelten konstruktiven Stil möglichst zu verbreiten. Das allerdings setzt ein vitales Festhalten an der Positivität der Marginalität voraus, das manchen in der Außenseiterposition abhanden zu kommen scheint. Es mag verständlich sein, dass wiederholte Niederlagen beim Kampf um die Ressourcen in der Wissenschaft irgendwann zu Resignation und Verbitterung führen können. Aber das Gefühl, sich zumindest als Märtyrer gegenüber dem unfairen, übermächtigen Mainstream zu fühlen, ist eine Form von sekundärem Krankheitsgewinn, der - als dritter Grund für den Änderungsunwillen - die eigene Irrelevanz nicht nur zementiert, sondern auch noch die Hauptverantwortlichkeit für deren Fortbestehen auf sich lädt. [10]

\section{Wege aus der selbstverschuldeten Irrelevanz}

\subsection{Integrativer Theorienpluralismus}

Der Weg aus der - in großen Teilen - selbstverschuldeten Irrelevanz des qualitativen Paradigmas führt also nur über die konstruktive Elaboration einer positiven Marginalität, die sich nicht scheut, in Kommunikation mit dem Mainstream zu treten, d.h. sich so weit anzupassen, dass man verstanden werden kann, ohne sich dabei aufzugeben, sich selbstaufhebend zu verbiegen. Wie soll das gehen? Der erste Schritt, im Sinn einer notwendigen Bedingung, ist aus meiner Sicht, eine andere psychische Einstellung zur MainstreamPsychologie zu entwickeln. Denn bei den meisten Vertretern/innen des qualitativen Paradigmas besteht diese Einstellung nach meiner Beobachtung in der Grundüberzeugung, dass "ihre" Psychologie die bessere ist. - Manche scheinen sogar zu glauben, dass qualitative Psychologen/innen die 'besseren Menschen' sind. - Ich will an dieser Stelle nicht ausführlich ontologisch, anthropologisch, ethisch und wissenschaftstheoretisch gegen diese Überzeugung argumentieren, sondern ganz pragmatisch auf einen Umstand hinweisen, der eigentlich allen Betroffenen die Freude an dieser Selbstaufwertung vergällen sollte: Es ist dies nämlich die Grundeinstellung, von der auch und gerade der Mainstream zutiefst überzeugt ist! Das ist ja die Rechtfertigungsgrundlage für alle Hegemonialität, für alle Marginalisierungsversuche gegenüber dem qualitativen Paradigma, dass letzteres aus der Sicht des quantitativen Paradigmas die 'schlechtere Psychologie' treibt. Dadurch, dass beide Seiten diese 
Grundüberzeugung von der prinzipiellen Überlegenheit des eigenen Ansatzes haben, kann sich in Bezug auf die Zusammenführung der beiden Traditionen nichts oder kaum etwas bewegen. In einer solch verfahrenen Situation sind die Klügeren gefragt, nicht, um nachzugeben, sondern um sich geistig zu bewegen: die Einstellung zu entwickeln, dass die Bandbreite und Komplexität des Menschen als Erkenntnis-Objekt so groß sind, dass beide Traditionen sinnvolle Arbeit leisten (können) (vgl. ERB 1997; GROEBEN 1986). Diese (zunächst einmal menschlich-subjektive) Grundeinstellung muss sich dann natürlich auch in wissenschaftlich-metatheoretischen Positionen abbilden. [11]

Die hier relevante metatheoretische Dimension ist die der Modellierung von Erkenntnisfortschritt, von Wissenschaftsentwicklung. Und da entspricht die Grundüberzeugung, die 'bessere Psychologie' zu betreiben, auf der Seite des Offstreams natürlich dem Revolutionsmodell des Erkenntnisfortschritts. Irgendwann, in naher oder ferner Zukunft, wird das herrschende Paradigma, das wissen wir seit KUHN (1967), untergehen und dann wird sich die Zuneigung der Geschichte 'unserem' Ansatz zuwenden - das ist die Hoffnung derer, die sich zumindest implizit als Revolutionäre des Faches sehen (möchten). Aber wie die historiographischen Analysen gezeigt haben, wird der Untergang eines herrschenden Paradigmas nicht von außen bewirkt, sondern nur von innen aufgrund der immer präziseren Identifikation der Anomien dieses Ansatzes durch inn selbst (o.c.; vgl. auch GROEBEN 1999). Die Hoffnung auf die Revolution bedeutet für die Außenseiter-Position also nur ein - relativ hilfloses - Abwarten. Dem Revolutionsmodell des Erkenntnisfortschritts anzuhängen, heißt daher in der gegenwärtigen Situation des qualitativen Paradigmas wiederum, die eigene Irrelevanz selbst mit zu verschulden. Erst das Gegenmodell einer reformerischen Fortentwicklung beider Paradigmen eröffnet dem Offstream wirksame Handlungsmöglichkeiten. Das muss nun keineswegs die konstruktive Ausarbeitung des eigenen Ansatzes als Chance einer positiven Marginalität abschneiden; ein bisschen Rebellion ist gut, nur darf sie nicht die Kommunikations- und Integrationsfähigkeit mit dem Mainstream aufheben. Der qualitative Ansatz wird, so also meine These, aus der selbst (mit) verschuldeten Irrelevanz nur herauskommen, wenn er sich der Reform der gesamten Psychologie widmet, nicht nur der Reform des quantitativen Paradigmas, sondern auch der eigenen Reform in dem Bemühen um eine konstruktive Verbindung mit der quantitativen Wissenschafts- und Methodenkonzeption (vgl. GROEBEN 1997, 2003). [12]

Dieses reformerische Integrationsbemühen muss also beiden Traditionen im Optimalfall komplementäre Aufgaben, Funktionen, Bereiche in einer den gesamten psychologischen Gegenstand umfassenden Wissenschaftskonzeption zuordnen. Auf höchstem Abstraktionsniveau impliziert das, entsprechend den eingangs diskutierten Ausgangsproblemen, eine möglichst gleichgewichtige Gegenstands-Methodik-Interaktion (so schon GROEBEN \& WESTMEYER 1981). Die Konzeptualisierung dieses Gleichgewichts ist letztlich, wie postuliert, nur im konstruktiven Austausch zwischen beiden Paradigmen möglich. Deshalb kann ich jetzt nur noch einige Stichworte für vorbereitende Schritte zu einer Binnenstrukturierung der Gegenstands- und Methodikdimension anbringen. In 
der Gegenstandsdimension bietet es sich an, die aktuellen Perspektiven einer Schichtenontologie auf- und auszuarbeiten (vgl. ALISCH 1993; 1995; GROEBEN 1999). Die neuere Diskussion zum Leib-Seele-Problem hat u.a. zu einer Stärkung des Emergenz-Konzepts geführt (vgl. z.B. HÖLSCHER 1997), das letztlich eine schichtenontologische Konzeptualisierung darstellt. Wenn man nämlich davon ausgeht, dass sich auf der Basis bestimmter komplexer neurophysiologischer Prozesse emergente mentale Prozesse beschreiben lassen, dann stellt das zumindest für die wissenschaftliche Theoriebildung eine ontologische Schichtung dar. Und diese Schichtung lässt sich weiter aufsteigend hierarchisieren in Richtung auf Handlungseinheiten, soziale Interaktion, soziale Strukturen und Institutionen etc. (was keine Beschränkung auf eine Substanz-Ontologie, sondern die Einbeziehung einer Prozess-Ontologie darstellt, vgl. RESCHER 1996; SEIBT 2003). Dabei steht die Psychologie im Mittelpunkt mit interdisziplinären Bezügen sowohl zu den fundierenden materialen Schichten des Mentalen, die vor allem von der quantitativ-naturwissenschaftlichen Tradition thematisiert und bearbeitet werden, als auch den komplexeren sozial-sinnorientierten Schichten, auf die traditionell der qualitative Ansatz eher ausgerichtet ist. Methodologisch impliziert das im Übrigen auch eine Verbindung von methodologischem Individualismus und Kollektivismus zu integrativen Mehrebenen-Modellen (GROEBEN 1999; MERAN 1979; VORDERER \& VALSINER 1999). Hier scheint mir relativ einfach eine Arbeitsteilung zwischen quantitativem Hauptstrom und qualitativen Nebenströmungen möglich im Sinne einer schichtenontologischen Schwerpunktsetzung, für die "lediglich" die emergenz-konzeptuelle Verbindung in der Mitte dieser Schichten-Hierarchisierung ausgearbeitet werden muss. Voraussetzung ist natürlich, dass beide Seiten die jeweils komplementären Gegenstandsdimensionen des psychologischen Erkenntnis-Objekts anerkennen: der qualitative Ansatz die Organismushaftigkeit des Menschen und der quantitative seine Sozialität und Sinngenerierungs-Kompetenz. Das allerdings erscheint mir ein erreichbares Ziel. [13]

\subsection{Integrative qualitativ-quantitative Versuchsplanung}

Schwieriger wird es m.E. in der Methodik-Dimension. Ganz grundsätzlich müsste hier aus meiner Sicht, wie schon angeführt, das qualitative Paradigma irgendeinen Konsens über die Außengrenzen des Methoden-Begriffs erarbeiten. Was kann, soll noch als Methodik anerkannt werden, was nicht; welche Intersubjektivitäts- und Systematikanforderungen sind zu stellen? Und diese Grenzziehung sollte, müsste m.E. durchaus auch unter Rückbezug auf die Vermittelbarkeit im quantitativen Ansatz diskutiert werden. Was nicht heißt, die quantitativen Kriterien letztlich den qualitativen überzuordnen oder überzustülpen (wie häufig von qualitativer Seite befürchtet wird: vgl. z.B. FLICK 1991a, b); andererseits, das was im quantitativen Ansatz über Jahrzehnte hinweg erarbeitet worden ist, kann ja nicht alles nur Unsinn sein. Von daher wäre z.B. schon zu überlegen, wie man mit der Allergie der 'Quantitativen' gegenüber Kunstlehren umgehen möchte. Ist es wirklich sinnvoll, quantitative Systematisierungsregeln relativ radikal dadurch zu konterkarieren, dass qualitative Verfahren als eine Art Kunst expliziert werden, in die man langsam hineinwachsen muss und für die es keine von jedermann und -frau anwendbaren Beurteilungskriterien gibt (vgl. z.B. 
die objektive Hermeneutik oder die Morphologische Psychologie)? Sicher sollte die Vielfalt und Flexibilität qualitativer Methodik nicht zugunsten einer (kurzschlüssigen) Anpassung an das quantitative Paradigma aufgegeben werden, aber rechtfertigt die Furcht vor solcher selbst beschneidenden Anpassung die Tolerierung aller Varianten, die auf anders Sozialisierte den Eindruck von Esoterik machen (müssen)? Hier einen Konsens innerhalb des qualitativen Ansatzes zu erzielen, der gleichzeitig als konstruktiver Ausgangspunkt für die Diskussion mit dem quantitativen Mainstream funktionieren kann, ist sicherlich ein sehr langfristiges Ziel. [14]

Es gibt aber durchaus auch kurz- und mittelfristige Möglichkeiten zur Elaboration der qualitativen Methodologie, die zum Ausgang aus der selbst verschuldeten Irrelevanz beitragen können. Die wichtigste kurzfristige Möglichkeit sehe ich in dem Ausbau und der deutlicheren Darstellung von Varianten qualitativer Versuchsplanung. Qualitative Methodologie ist - auch im Bewusstsein ihrer Verfechter/innen - noch viel zu sehr auf die Perspektive von (qualitativen) Erhebungsmethoden eingeschränkt. Dabei gehört die Versuchsplanung sicher genauso gewichtig zum qualitativen Ansatz, zumal es im Bereich qualitativer Untersuchungsdesigns wichtige Extensionen dessen gibt, was innerhalb des quantitativen Ansatzes als Ergänzung zum Königsweg des Experiments in Form von quasi-experimentellen Versuchsplänen erarbeitet worden ist. Solche qualitativen Erweiterungen auch der quasi-experimentellen Versuchsplanung reichen von der bewussten Stichprobenziehung bis zur Fallstudie (als Einzelfalloder multiple Fallstudie: vgl. SCHREIER 2006). Es gibt aus spezifischen Forschungsprogrammen stammende spezielle Untersuchungssequenzierungen (vgl. die Gegenstandsbezogene Theoriebildung, das Forschungsprogramm Subjektive Theorien und die Ethnographie; o.c.) und vor allem sog. Mixed Methods- und Mixed Model-Designs, die selbst schon eine direkte Realisierung von Integrationsperspektiven zwischen qualitativem und quantitativem Paradigma darstellen (o.c. und TASHAKKORI \& TEDDLIE 1998). Hier sollte es ein Leichtes sein, die von TASHAKKORI und TEDDLIE vorgenommene Strukturierung in zeitlicher und hierarchischer Perspektive in absehbarer Zeit noch weiter systematisch mit prototypischen Realisierungsvarianten auszuarbeiten: also z.B. sequenzielle Varianten des Einsatzes von zuerst qualitativen, dann quantitativen (Erhebungs-) Methoden und vor allem auch in der umgekehrten Reihenfolge; parallele Designs und insbesondere auch hybride, in denen qualitative und quantitative Anteile bereits in der Beobachtungsmethodik integriert sind. Und die hierarchisierende Perspektive sollte ausgearbeitet werden nicht nur hinsichtlich gleichberechtigter Gewichtungen von quantitativen und qualitativen Anteilen, sondern auch und gerade bezüglich Ansätzen, bei denen die eine Seite das Übergewicht hat, übergeordnet ist. Solche, auch kurzfristig leistbaren, Ausdifferenzierungen einer qualitativen Versuchsplanung böten die Grundlage dafür, das qualitative Paradigma aus der Ecke einer Defizitvariante von Methodologie herauszubringen, in der es immer nur um das Problem der Erhebung subjektiver Erlebensaspekte geht (die für die Intersubjektivität des wissenschaftlichen Erkenntnisprozesses insgesamt als störend oder beschädigend angesehen werden). Dabei scheint es die qualitative Methodologie in anderen Sozialwissenschaften im Kontrast zur Psychologie z.T. schon geschafft 
zu haben, diese 'Defizitecke' zu verlassen; hier wäre nach der Übertragbarkeit der dabei eingesetzten Strategien auf die Psychologie zu fragen. [15]

\subsection{Ausarbeitung komplexer statistischer Auswertungsansätze}

Für alle sozialwissenschaftlichen Disziplinen aber sehe ich den wichtigsten Ansatzpunkt zum Ausgang aus der selbst verschuldeten Irrelevanz des qualitativen Ansatzes in der mittelfristig zu leistenden Auflösung eines völlig unsinnigen Fremd-, z.T. aber auch wieder Selbststereotyps. Es ist dies die Implikation, dass qualitative Forschung und quantitativ-statistische Auswertung nicht zusammenpassen, nicht zusammengehen. Das Fremdstereotyp besteht in extremster Ausprägung in dem Verdacht, dass qualitativ Forschende deshalb dieses Paradigma gewählt haben, weil sie keine Statistik beherrschen. Das Selbststereotyp geht von einer inhärenten Spannung, Gegenläufigkeit zwischen qualitativ aussagekräftigen (subjektiv-individuellen) Daten und differenzierten statistischen Auswertungsansätzen aus. Damit aber haben sich die qualitativ Forschenden von einer wissenschaftstheoretischen Fehlklassifikation und -interpretation infizieren lassen. Im quantitativen Paradigma ist immer von statistischen Auswertungsmethoden die Rede, und die Statistik stellt einen Löwenanteil der Methodikausbildung dar. Dies ist jedoch ein wissenschaftstheoretisch inadäquater, irreführender Begriffsgebrauch von Methodik. Wenn man unter 'Methoden' systematische Verfahren versteht, mit denen am psychologischen Gegenstand bestimmte (theoriegeleitet intendierte) Merkmale abgehoben werden, dann sind statistische Auswertungsverfahren keine 'Methoden'. Vielmehr ist es so, dass zum Zeitpunkt, an dem im Forschungsprozess statistische Auswertungsansätze zur Anwendung kommen, die zentralen, nämlich gegenstandskonstituierenden Methodikteilmengen längst gelaufen sind. Insofern gibt es auch keinerlei sachlogisch begründete Gegenläufigkeit oder sogar Ausschließung von qualitativer Methodik und statistischer Auswertungsprozedur. Das Gegenteil ist m.E. der Fall. Im qualitativen Ansatz wird eine solche Fülle von (eben auch idiographischen) Daten generiert, dass besonders differenzierte, sophistizierte statistische Auswertungsmodelle vonnöten sind, um zu aussagekräftigen Interpretationen zu gelangen. Und komplementär: Der quantitative Ansatz arbeitet mit möglichst so standardisierten Versuchsplänen und Datenerhebungsprozeduren, dass hier Standardvarianten von statistischen Auswertungsmodellen in der Regel völlig ausreichen. Das qualitative Paradigma, so meine These, ist diejenige Forschungstradition, die die größere Kohärenz zu differenzierter statistischer Auswertung aufweist, zur Adaption und Weiterentwicklung komplexer, sophistizierter Auswertungsmodelle. [16]

Das beginnt z.B. mit Auswertungsansätzen, die die oben angeführte schichtenontologische Komplexität des Menschen als sozialem Wesen einschließlich der Integration des methodologischen Individualismus und Kollektivismus zu Mehrebenenmodellen widerspiegeln. Als soziales Subjekt ist das psychologische Erkenntnis-Objekt immer Teil von größeren sozialen Einheiten, wie z.B. Peer groups, religiösen oder ethnisch-kulturellen Gemeinschaften, Schicht- oder Lebensstilebenen, politische 
Gesellschaftsstrukturen etc. Um solche in der Datenstruktur enthaltenen Schichtungsebenen auswertungstechnisch adäquat abzubilden, sind mehrebenenanalytische Auswertungsmodelle einzusetzen (vgl. HUININK 1989; LANGER 2004), die auch im quantitativen Paradigma noch viel zu selten zur Anwendung kommen. Dabei ist in der Regel von komplexen Beziehungen zwischen den unterschiedlichen Schichten, z.B. der Individual- und Kollektivebene, im Sinne dynamischer Interaktionsprozesse auszugehen (vgl. BOKER 2002). Dadurch sind die üblichen multivariaten Verfahren, bei denen Eigenschaften starr zueinander in Beziehung gesetzt werden, überfordert, weil die statistische Analyse die Veränderungsdynamik eines Systems über die Zeit hinweg abbilden können muss, wie es z.B. bei der Funktionalen Datenanalyse der Fall ist (vgl. RAMSAY \& SILVERMAN 2005). Ein typisches Beispiel ist das Konstrukt der Ko-Konstruktion (vgl. GROEBEN 2005; YOUNISS 1994) für die individuelle Kompetenzentwicklung, z.B. bei der Lesesozialisation. Die jeweils aktuellen individuellen Entwicklungsmöglichkeiten werden von den in sozialen Normen enthaltenen Mitgliedschaftsentwürfen der Gesellschaft einerseits beschränkt, tragen andererseits aber auch zur Veränderung von gesellschaftlichen Strukturen in Peer-groups, Schulklassen etc. bei, wodurch sich wieder für das Individuum neue Entwicklungspotenziale ergeben. Solche dynamischen Prozesse (vgl. RESCHER 1996; SEIBT 2003) sind in der Regel nicht durch lineare Interaktionen der Einzelelemente abzubilden, sondern implizieren nichtvorhersehbare, chaotische Prozessbeziehungen, die durch nicht-lineare Zeitreihenanalysen (vgl. ALISCH 2001) erfasst werden (können). [17]

Ein paralleler Ansatzpunkt betrifft die Vielschichtigkeit von Sinnstrukturen, wie sie bei der qualitativen Erhebung semantischer Informationen meistens vorliegen und ausgewertet werden müssen (von Interview-Transkriptionen bis zu Subjektiven Theorie-Strukturen). Die klassische Variante der Inhaltsanalyse ist hier in der Regel nur in der Lage, einen geringen Anteil der im Material enthaltenen Informationen aufzuarbeiten. Quantitative Weiterentwicklungen wie das "Data-Mining" oder "Text-Mining" (vgl. WEISS, INDURKHYA, ZHANG \& DAMERAU 2005) bringen die semantischen Informationen in ein quantifizierbares Format, auf das verschiedene Formen statistischer Lernalgorithmen angewandt werden (vgl. WALLACE 2005). Dadurch ist es möglich, z.B. typische Denkstrukturen in Texten zu identifizieren wie Unterschiede in Erlebnisberichten zwischen Kindern und Erwachsenen, die Differenziertheit oder Mehrdimensionalität der Kriegsberichterstattung über den Irak-Krieg im Vergleich von USA und Europa etc. [18]

Dies sind nur einige Beispiele für komplexe statistische Analysen qualitativer Daten, die durch weitere Ansätze zur Aufarbeitung semantischer Strukturen in Netzwerk-Konzepten etc. (vgl. OLDENBÜRGER, 2005) ergänzt werden können. [19]

Es gibt also aus meiner Sicht durchaus Wege aus der selbst verschuldeten Irrelevanz des qualitativen Offstreams. Sie bestehen kurz- und mittelfristig in der Ausarbeitung der qualitativen Untersuchungsplanung und der Elaboration komplexer, sophistizierter statistischer Auswertungsmodelle - Elaborationen, die dann offensiv-konstruktiv in einen integrationsorientierten Austausch mit dem 
quantitativen Paradigma einzubringen sind. Das ist die Entwicklung, die ich mir vorstelle ... Das ist die Phantasie, die ich habe: Die Gate keeper der Forschungsförderung (in der DFG oder sonstwo) sitzen über einem hochkomplexen, differenzierten Projektantrag aus dem Bereich praxisrelevanter Forschung, und die einhellige Meinung ist: Da brauchen wir ein Sondergutachten aus der qualitativen Ecke, um zu sichern, dass wir uns mit der (qualitativen) Versuchsplanung und der geplanten statistischen Auswertung nicht interdisziplinär und international blamieren ... Von Ihnen (den Jüngeren) wird es abhängen, ob sich diese Phantasie als eine realisierbare Utopie oder als unrealistische Illusion erweisen wird ... [20]

\section{Danksagung}

Ich danke L. M. ALISCH und S. SCHROEDER für Beratung bei der Problematik der statistischen Auswertungsmodelle.

\section{Literatur}

Alisch, Lutz-Michael (1993). Probleme der Rekonstruktion psychologischer Handlungstheorien II. Mikro-Makro-Problem, lokale und gekoppelte Dynamik, Kernstruktur. grkg/Humankybernetik, 34, 68-77.

Alisch, Lutz-Michael (1995). Pädagogische Wissenschaftslehre. Zum Verhältnis von Ethik, Psychologie und Erziehung. Münster: Waxmann.

Alisch, Lutz-Michael (Hrsg.) (2001). Nicht-lineare Zeitreihenanalyse [Themenheft]. Empirische Pädagogik, 15(1).

Boker, Steven M. (2002). Consequences of continuity: The hunt for intrinsic properties within parameters of dynamics in psychological processes. Multivariate Behavioral Research, 37, 405422.

Erb, Egon (1997). Gegenstands- und Problemkonstituierung: Subjekt-Modelle (in) der Psychologie. In Norbert Groeben (Hrsg.), Zur Programmatik einer sozialwissenschaftlichen Psychologie, Band I Metatheoretische Perspektiven, 1. Halbband Gegenstandsverständnis, Menschenbilder, Methodologie und Ethik (S.139-239). Münster: Aschendorff.

Flick, Uwe (1991a). Stationen des qualitativen Forschungsprozesses. In Uwe Flick, Ernst von Kardorff, Heiner Keupp, Lutz von Rosenstiel \& Stephan Wolff (Hrsg.), Handbuch Qualitative Sozialforschung. Grundlagen, Konzepte, Methoden und Anwendungen (S.148-173). München: Psychologie Verlags Union.

Flick, Uwe (1991b). Triangulation. In Uwe Flick, Ernst von Kardorff, Heiner Keupp, Lutz von Rosenstiel \& Stephan Wolff (Hrsg.), Handbuch Qualitative Sozialforschung. Grundlagen, Konzepte, Methoden und Anwendungen (S.432-434). München: Psychologie Verlags Union.

Flick, Uwe, Kardorff, Ernst, Keupp, Heiner von, Rosenstiel, Lutz von \& Wolff, Stephan (Hrsg.) (1991). Handbuch Qualitative Sozialforschung. Grundlagen, Konzepte, Methoden und Anwendungen. München: Psychologie Verlags Union.

Franck, Robert (Hrsg.) (2002). The explanatory power of models. Dordrecht: Kluwer.

Fröhlich, Gerhard (1999). Das Messen des leicht Meßbaren. Output-Indikatoren, Impact-Maße: Artefakte der Szientometrie? In Jörg Becker \& Wolf Göhring (Hrsg.), Kommunikation statt Markt. Zu einer alternativen Theorie der Informationsgesellschaft (S.27-38). Sankt Augustin: GMDForschungszentrum.

Geuter, Ulfried (1986). Psychologie im Nationalsozialismus. In Günter Rexelius \& Siegfried Grubitzsch (Hrsg.), Psychologie. Theorien - Methoden - Arbeitsfelder. Ein Grundkurs (S.557-598). Reinbek: Rowohlt.

Graumann, Carl Friedrich (1985). Psychologie im Nationalsozialismus. Berlin: Springer. 
Groeben, Norbert (1997). Einleitung: Sozialwissenschaftliche Psychologie-Konzeption zwischen Natur- und Geisteswissenschaft. In Norbert Groeben (Hrsg.), Zur Programmatik einer sozialwissenschaftlichen Psychologie, Band I Metatheoretische Perspektiven, 1. Halbband Gegenstandsverständnis, Menschenbilder, Methodologie und Ethik (S.1-26). Münster: Aschendorff.

Groeben, Norbert (1999). Fazit: Die metatheoretischen Merkmale einer sozialwissenschaftlichen Psychologie. In Norbert Groeben (Hrsg.), Zur Programmatik einer sozialwissenschaftlichen Psychologie, Band I Metatheoretische Perspektiven, 2. Halbband Theoriehistorie, Praxisrelevanz, Interdisziplinarität, Methodenintegration (S.311-404). Münster: Aschendorff.

Groeben, Norbert (2003). Fazit: Problemaufriss einer Theoretischen Psychologie. In Norbert Groeben (Hrsg.), Zur Programmatik einer sozialwissenschaftlichen Psychologie, Band II Objekttheoretische Perspektiven, 2. Halbband Situationsbezug, Reflexivität, Rationalität, Theorieintegration (S 317-421). Münster: Aschendorff.

Groeben, Norbert (2005). (Lese-)Sozialisation als Ko-Konstruktion - Methodisch-methodologische Problemlösungsperspektiven. In Norbert Groeben \& Bettina Hurrelmann (Hrsg.), Lesesozialisation in der Mediengesellschaft. Ein Forschungsüberblick (S.145-168). Weinheim: Juventa.

Groeben, Norbert \& Westmeyer, Hans (1981). Kriterien psychologischer Forschung. München: Juventa.

Grünberg, Adolf (1988). Die Grundlagen der Psychoanalyse - Eine philosophische Kritik. Stuttgart: reclam.

Herrmann, Theo (1994). Forschungsprogramme. In Theo Herrmann \& Werner Tack (Hrsg.), Enzyklopädie der Psychologie: Themenbereich Methodologische Grundlagen der Psychologie, Serie Forschungsmethoden der Psychologie, Band 1 (S.251-294). Göttingen: Hogrefe.

Hölscher, Stefan (1997). Monismus und Dualismus in der Psychologie. Zur Verschränktheit von ontologischen und methodologischen Problem(lösungs)perspektiven. In Norbert Groeben (Hrsg.), Zur Programmatik einer sozialwissenschaftlichen Psychologie, Band I Metatheoretische Perspektiven, 1. Halbband Gegenstandsverständnis, Menschenbilder, Methodologie und Ethik (S.27-138). Münster: Aschendorff.

Huinink, Johannes (1989). Mehrebenensystem-Modelle in den Sozialwissenschaften. Wiesbaden: Deutscher Universitätsverlag.

Jüttemann, Gerd (Hrsg.). (1983). Psychologie in der Veränderung. Perspektiven für eine gegenstandsangemessene Forschungspraxis. Weinheim: Beltz.

Jüttemann Gerd (1992). Psyche und Subjekt. Für eine Psychologie jenseits von Dogma und Mythos. Reinbek: Rowohlt.

König, Eckhard \& Zedler, Peter (1995). Bilanz qualitativer Forschung. Band I Grundlagen qualitativer Forschung. Weinheim: Deutscher Studien Verlag.

Kuhn, Thomas S. (1967). Die Struktur wissenschaftlicher Revolutionen. Frankfurt/M.: Suhrkamp.

Kuhn, Thomas S. (1972). Postscript - 1969, Zur Analyse der Struktur wissenschaftlicher Revolutionen. In Peter Weingart (Hrsg.), Wissenschaftssoziologie 1: Wissenschaftliche Entwicklung als sozialer Prozeß (S.287-319). Frankfurt a.M.: Suhrkamp.

Langer, Wolfgang (2004). Mehrebenenanalyse: eine Einführung für Forschung und Praxis. Wiesbaden: VS Verlag für Sozialwissenschaften.

Lamnek, Siegfried (1988). Qualitative Sozialforschung. Band 1 Methodologie. München: Psychologie Verlags Union.

Lamnek, Siegfried (1989). Qualitative Sozialforschung. Band 2 Methoden und Techniken. München: Psychologie Verlags Union.

Laucken, Uwe (1989). Denkformen der Psychologie dargestellt am Entwurf einer Logographie der Gefühle. Bern: Huber.

Leuninger, Helen (1993). Reden ist Schweigen, Silber ist Gold. München: dtv.

Leuninger, Helen (1996). Danke und Tschüß fürs Mitnehmen. Zürich: Ammann.

Lück, Helmut E. (1996). Geschichte der Psychologie: Strömungen, Schulen, Entwicklungen. Stuttgart: Kohlhammer.

Meran, Josef (1979). Individualismus oder Kollektivismus? Zeitschrift für allgemeine Wissenschaftstheorie, $X(1), 35-53$. 
Métraux, Alexandre (1985). Der Methodenstreit und die Amerikanisierung der Psychologie in der Bundesrepublik 1950-1970. In Mitchell G. Ash \& Ulfried Geuter (Hrsg.), Geschichte der deutschen Psychologie im 20. Jahrhundert (S.225-252). Opladen: Westdeutscher Verlag.

Oldenbürger, Hartmut-A. (2005). Netz-Werk-Zeuge. R-Programme zur Analyse semantischer Strukturdaten. Version 1.93. Verfügbar unter: http://www.liteline.de/ holdenb/fst/nwz/nwz-prog.html [Letzter Zugriff: 23.6.2006].

Ramsay, James O. \& Silverman, Bernard W. (2005). Functional data analysis (2. Auflage). New York: Springer.

Rescher, Nicholas (1996). Process metaphysics. An introduction to process philosophy. New York: University Press.

Schreier, Margrit (2006). Qualitatives Untersuchungsdesign. In Norbert Groeben \& Bettina Hurrelmann (Hrsg.), Empirische Unterrichtsforschung in der Literatur- und Lesedidaktik (S.338354). Weinheim: Juventa.

Schurz, Gerhard (Hrsg.) (1988). Erklären und Verstehen in der Wissenschaft. München: Oldenbourg.

Seibt, Johanna (Hrsg.) (2003). Process theories: Crossdisciplinary studies in dynamic categories. Dordrecht: Kluwer.

Tashakkori, Abbas \& Teddlie, Charles (1998). Mixed methodology. Combining qualitative and quantitative approaches. Thousand Oaks, CA: Sage.

Toulmin, Stephen \& Leary, David E. (1985). The cult of empiricism in psychology, and beyond. In Sigmund Koch \& David E. Leary (Hrsg.), A century of psychology as science (S.594-617). New York: McGraw-Hill.

Unger, Rhoda K. (2000). Outsiders inside: Positive marginality and social change. Journal of Social Issues, 56(1), 163-179.

Vorderer, Peter \& Valsiner, Jan (1999). (Sozial-)Psychologie und Soziologie - Oder: Das MikroMakro-Problem(-Bewußtsein). In Norbert Groeben (Hrsg.), Zur Programmatik einer sozialwissenschaftlichen Psychologie, Band I Metatheoretische Perspektiven, 2. Halbband Theoriehistorie, Praxisrelevanz, Interdisziplinarität, Methodenintegration (S.311-404). Münster: Aschendorff.

Wallace, Christopher S. (2005). Statistical and inductive inference by Minimum Message Length. New York: Springer.

Weingart, Peter (Hrsg.) (1972). Wissenschaftssoziologie 1. Wissenschaftliche Entwicklung als sozialer Prozess. Frankfurt/M.: Athenäum Fischer.

Weiss, Sholom M.; Indurkhya, Nitin; Zhang, Tong \& Damerau, Fred J. (2005). Text mining. Predictive methods for analyzing unstructured information. New York: Springer.

Youniss, James (1994). Soziale Konstruktion und psychische Entwicklung. Frankfurt/M.: Suhrkamp. 


\section{Zum Autor}

Norbert GROEBEN, Univ.-Prof. (Allgemeine Psychologie und Kulturpsychologie); Universität Köln; Arbeitsschwerpunkte: Wissenschaftstheorie und (Qualitative) Methodologie; Sprach- und Denkpsychologie; Theoretische Psychologie und Psychologische Anthropologie; Empirische Literaturwissenschaft (Hon.Prof. f. Allg. u. Emp. Lit.wiss., Universität Mannheim)
Kontakt:

Prof. Dr. N. Groeben

Psychologisches Institut der Universität zu Köln, Lehrstuhl II: Allgemeine Psychologie und Kulturpsychologie

Herbert-Lewin-Str. 2

D-50931 Köln

E-Mail: n.groeben@uni-koeln.de URL: http://www.uni-koeln.de/philfak/psych/allgemeine/index.html

\section{Zitation}

Groeben, Norbert (2006). Gibt es Wege aus der selbstverschuldeten Irrelevanz des qualitativen Offstreams? [20 Absätze]. Forum Qualitative Sozialforschung / Forum: Qualitative Social Research, 7(4), Art. 34, http://nbn-resolving.de/urn:nbn:de:0114-fqs0604347. 